\title{
A Passive Anti-icing Strategy Based on a Superhydrophobic Mesh with Extremely Low Ice Adhesion Strength
}

\author{
Peng Wang ${ }^{12^{*}}$, Ziqiang $\mathrm{Li}^{1,2}$, Qing Xie ${ }^{3}$, Wei Duan ${ }^{1,2}$, Xinchun Zhang ${ }^{1,2}$, Huilong Han ${ }^{1,2}$ \\ 1. School of Energy, Power and Mechanical Engineering, North China Electric Power University, Baoding 071000, China \\ 2. Hebei Key Laboratory of Electric Machinery Health Maintenance \& Failure Prevention, North China Electric Power University, \\ Baoding 071003, China \\ 3. School of Electrical and Electronic Engineering, North China Electric Power University, Baoding 071003, China
}

\begin{abstract}
Although superhydrophobic materials have attracted much research interest in anti-icing, some controversy still exists. In this research, we report a cost-effective method used to verify the contribution of area fraction to ice adhesion strength. We tried to partially-embed silica nanoparticles into microscale fabrics of a commercial polyamide mesh. Then, the area fraction could be determined by altering the mesh size. Generally, the ice adhesion strength decreases as the area fraction decreases. An ice adhesion strength of $\sim 1.9 \mathrm{kPa}$ and a delayed freezing time of $\sim 1048 \mathrm{~s}$ can be obtained. We attribute the low ice adhesion strength to the combination of superhydrophobicity and stress concentration. The superhydrophobicity prohibits the water from penetrating into the voids of the meshes, and the small actual contact area leads to stress concentration which promotes interfacial crack propagation. Moreover, our superhydrophobic mesh simultaneously exhibits a micro-nano hierarchical structure and a partially-embedded structure. Therefore, the as-prepared superhydrophobic mesh retained the icephobicity after $20 \mathrm{icing} /$ deicing cycles, and maintained its superhydrophobicity even after 60 sandpaper-abrasion cycles and a $220{ }^{\circ} \mathrm{C}$ thermal treatment.
\end{abstract}

Keywords: bionic, superhydrophobic, anti-icing, mechanical robustness, hierarchical structure, partially-embedded structure Copyright $(C)$ The author(s) 2021.

\section{Introduction}

Ice accretion tends to cause serious problems even disasters in many areas, such as power transmission lines, aircrafts, and telecommunication networks ${ }^{[1-4]}$. Particularly, the longstanding freezing rain in 2008 led to a $37 \%$ $500 \mathrm{kV}$ transmission tower in South China to fall due to weight overloading ${ }^{[5]}$. To solve this problem, many methods have been developed for de-icing and anti-icing. Among these methods, superhydrophobic surfaces have attracted increasing attention as a potential avenue for eliminating ice accumulation ${ }^{[6]}$.

It is well known that the lotus leaf typically exhibits superhydrophobicity with water Contact Angles (CA) greater than $150^{\circ}$ and Sliding Angles (SA) less than $10^{\circ}$. Benefitting from the combination of the low surface energy and suitable morphology ${ }^{[7-10]}$, the water droplets can not stay on the surface of lotus leaves. Inspired by this phenomenon, many studies have been done to investigate the anti-icing performance of superhydro- phobic surfaces. It has been found that superhydrophobic surfaces have advantages in delaying freezing time, decreasing the adhesion force, or both ${ }^{[2-4]}$. However, most superhydrophobic surfaces demonstrated an adhesion strength larger than $100 \mathrm{kPa}$, which was much higher than that of slippery surfaces ${ }^{[11]}$. To solve this problem, Xie et al. prepared a superhydrophobic dual-scale structure, and obtained a low adhesion strength of $\sim 45 \mathrm{kPa}^{[12]}$. Pan et al. recently reported an extremely high Cassie-Baxter state by means of dual-scale micro-nano structure, but no ice adhesion was reported $^{[13]}$. Meanwhile, Ling et al. point out that suitable surface topographies could promote interfacial crack propagation, and then reduced ice adhesion ${ }^{[14]}$. Therefore, fabricating superhydrophobic materials with a suitable micro-nano structure may be a solution to reducing ice adhesion.

Furthermore, the lack of mechanical robustness has become a major challenge that prohibits the practical application of superhydrophobic materials. Kulinich et al.

\footnotetext{
*Corresponding author: Peng Wang

E-mail: wangpeng1986@ncepu.edu.cn
} 
further claimed that the cyclic icing/deicing process would break the micro/nano structure of the superhydrophobic surfaces and then dramatically reduce the icephobicity ${ }^{[15]}$. Many efforts have been made to improve the mechanical durability. Recently, Parkin's group introduced a "Paint+Adhesive" method ${ }^{[16]}$. By bonding fluorinated $\mathrm{TiO}_{2}$ nanoparticles with commercial adhesives, the sample maintained superhydrophobicity even after $8.00 \mathrm{~m}$ abrasion under a $100 \mathrm{~g}$ load. Meanwhile, many researchers adapted hierarchical structures and tried to utilize the micro-protrusions to protect the fragile nanostructures ${ }^{[17]}$. In our previous work, we introduced a simple dissolution and resolidification method which could combine partially-embedded nanoparticles with hierarchical roughness ${ }^{[18]}$. Nevertheless, micro structure comes from uneven dissolution and re-solidification of the PS substrate, which is difficult to control.

Recently, Nguyen et al. fabricated a well-textured surface, and obtained a minimum ice adhesion of $\sim 3 \mathrm{kPa}$ by altering the areal fraction ${ }^{[19]}$. However, the well-textured surfaces were prepared by colloidal lithography and dry etching on a quartz glass surface, which is expensive and time-consuming. Moreover, no mechanical robustness was reported. Thus, how to achieve a suitable areal fraction and excellent mechanical robustness by a cheap and easy way is still a large challenge. From the viewpoint of large scale applications, a polyamide mesh has been utilized as the substrate to fabricate superhydrophobic materials ${ }^{[20]}$. Unlike other substrates, the areal fraction of a polyester mesh could be determined by altering the mesh size in the local market. In this research, we tried to partially-embed silica nanoparticles into the microscale polyamide fabrics of the mesh. Then, a dual-scale micro-nano structure with a tunable microscale size was obtained. The ice adhesion was further optimized by altering the parameters. The minimum ice adhesion strength can be as small as $1.9 \mathrm{kPa}$. Moreover, the icephobicity can be retained after $20 \mathrm{icing} /$ deicing cycles, and the superhydrophobicity can be maintained even after 60 sandpaper-abrasion cycles and a $220{ }^{\circ} \mathrm{C}$ thermal treatment.

\section{Experiments}

\subsection{Materials}

The polyamide meshes with different mesh sizes were purchased from Jiangsu Honghao Wire Mesh Co. Ltd, China. The hydrophobic silica nanoparticles (R812, $7 \mathrm{~nm}-40 \mathrm{~nm}$ in diameter) were purchased from Degussa. Formic acid ( $\geq 85 \%$ ) and ethanol ( $\geq 99 \%$ ) were purchased from Tianjin Kemiou Chemical Reagent Co., Ltd, China. All chemicals were analytical grade reagents and were used as received.

\subsection{Preparation of nanoparticle suspension}

First, $3.5 \mathrm{~g}$ of formic acid was added to $6.5 \mathrm{~g}$ of ethanol. After $10 \mathrm{~min}$ of stirring, $0.5 \mathrm{~g}$ of silica nanoparticles were put into the solution. The solution was further stirred for $20 \mathrm{~min}$ before use.

\subsection{Dissolution and re-solidifcation process used to prepare the superhydrophobic mesh}

First, $30 \mathrm{~mL}$ of the nanoparticle suspension was put into a container. Then, the polyamide mesh was immersed in the nanoparticle suspension. After sealing the container, the container was kept in a preheated oven at a constant temperature of $60{ }^{\circ} \mathrm{C}$ for $1 \mathrm{~h}$. Finally, the mesh was taken out of the nanoparticle suspension and dried in air.

\subsection{Characterization}

The surface microstructures of the superhydrophobic mesh were measured by Scanning Electron Microscopy (SEM, TESCAN Vega3). Before SEM, a thin Au film $(\sim 2 \mathrm{~nm}-3 \mathrm{~nm})$ was sputtered onto the samples. The $X$-ray Photoelectron Spectroscopy (XPS, Thermo ESCALAB 250XI, USA) was utilized to determine the chemical composition of the sample. A home-made contact angle meter was adapted to measure the water CAs. We further utilized a high speed camera (Revealer 2F04) to evaluate the SAs. Five microlitre water droplets were used in the aforementioned CA and SA test.

\subsection{Measurement of the ice adhesion strength}

A home-made device was utilized to measure the ice adhesion strength, as shown in Fig. S1 in the supplementary file. This device was built based on a previous method with some modifications ${ }^{[21,22]}$. A climate chamber (QL-HWHS-100, Xiamen Qunlong Sci-Tech Co., Ltd., China) was utilized to control the temperature $(-15$ ${ }^{\circ} \mathrm{C}$ ) and humidity (30\%). An infrared imager (FLIR-C2, 
FLIR Ltd., USA) was utilized to assess the surface temperature of the sample. Deionized water $(\sim 1 \mathrm{~mL})$ was dropped into glass column $(25 \mathrm{~mm} \times 25 \mathrm{~mm} \times 40 \mathrm{~mm})$, and then the glass column was loaded onto the sample by following the method shown in Ref. [21]. Deionized water was completely frozen after $6 \mathrm{~h}$. Then, a force transducer (Imada, model ZTS-500N) was mounted on the motion stage, and pushed the frozen ice column by controlling the motion stage. The maximum force needed to detach the ice column from the surface was further divided by the cross-section area of the ice, and the resulting value was defined as the ice adhesion strength.

\subsection{Measurement of the freezing time}

In this research, a Peltier cooler was utilized to control the temperature $\left(-15{ }^{\circ} \mathrm{C}\right)$. Then, a $5 \mu \mathrm{L}$ water droplet was carefully dropped onto the sample surface with the help of a syringe. A digital microscope (Andonstar-A1, China) was utilized to measure the freezing process.

\section{Results and discussion}

\subsection{Adjustable double-scale micro-nano superhy- drophobic mesh}

In this research, polyamide meshes with different mesh sizes (60-, 80-, 100-, 300-, 400-, and 500-mesh) were utilized as the substrate. The micro structures of the meshes were investigated by an optical microscope, as shown in Fig. S2. We further tried to partially embed the silica nanoparticles into the microscale polyamide fabrics of the mesh. Then, a double-scale micro-nano structure with an adjustable micro-scale could be obtained, as shown in Fig. 1. By altering the parameters, the area fraction of the surface can be determined.

Then, determining how to partially embed the silica nanoparticles into the PA mesh is crucial for preparing mechanically robust superhydrophobic materials. In our previous research, the dissolution and re-solidifcation method has been introduced ${ }^{[22]}$, and the key point was finding an appropriate solvent. It has been found that formic acid is a good solvent for polyamide. Thus, we tried to disperse the modified silica nanoparticles in formic acid in our experiment (Fig. 2). After immersing the PA meshes into the formic acid and heating at $60{ }^{\circ} \mathrm{C}$, formic acid tended to dissolve the PA, which let the interface between the PA and solvent become viscous. Then, the silica nanoparticles could get into the interface. After $1 \mathrm{~h}$ reaction, the PA mesh was pulled out with some solution adhering on it. After evaporating the solution, the surface of the PA mesh would be resolidificated again. In fact, the initial experimental result was totally different than our expectations. Pure formic acid dissolves the polyamide mesh so well that we can not find the mesh in the solution after the $1 \mathrm{~h}$ reaction. Thus, we tried to dilute formic acid using ethanol. After trial and error, the formic acid/ethanol solution (35: 65, w: w) was finally determined because it achieved the best anti-abrasion results.

\subsection{The characterization of the surface morphology and chemical composition}

To confirm the formation mechanism of the superhydrophobic mesh, SEM observation was performed and the 100-mesh superhydrophobic mesh was utilized as the sample (Figs. $3 a-3 c$ ). Many micro scale bulges could be found on the surface of the fabrics (Fig. 3b). The high magnification SEM image (Fig. 3c) further indicated that these micro bulges were composed of nanoparticles. We attributed this to the aggregation state of the silica nanoparticles in the ethanol/formic acid solution ${ }^{[23]}$. Besides surface roughness, the surface energy is also crucial for achieving superhydrophobicity. Then, the XPS tests were performed. As shown in Fig. S3, the survey spectrum consists of $\mathrm{Si} 2 \mathrm{p}, \mathrm{C} 1 \mathrm{~s}, \mathrm{O} 1 \mathrm{~s}$ and $\mathrm{N}$ 1s peaks. In this research, the commercial silica nanoparticles were modified by the hexamethyldisilazane, which could lower the surface energy even without a $\mathrm{F}$ component ${ }^{[24]}$. The combination of the surface roughness and low surface energy endows the as-prepared mesh with excellent superhydrophobicity, which can be confirmed from the droplet bouncing test (see supplementary movies $1-6$ ). As shown in Fig. 3d, we recorded a typical process of a droplet bouncing on the 100-mesh superhydrophobic surface. After the water droplet fell from a height of $\sim 3 \mathrm{~cm}$, it could bounce three times before leaving the surfaces. Moreover, a tiny satellite droplet (the dotted circle) appears during the first bouncing process. The tiny satellite droplet could also bounce off without wetting the surface. This excellent bouncing performance indicated that the water 
(a)

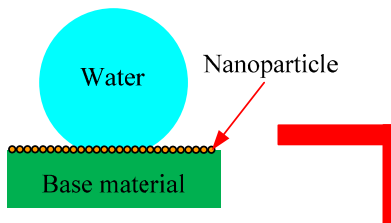

(b)

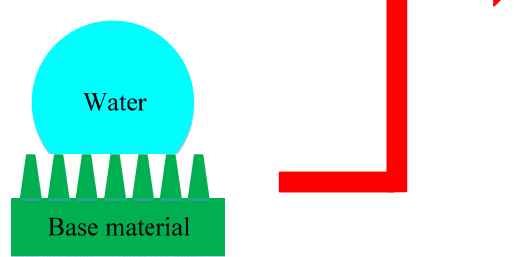

(c)

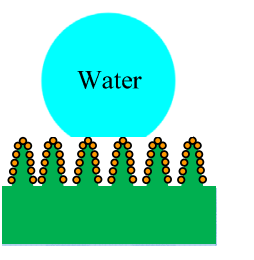

(d)

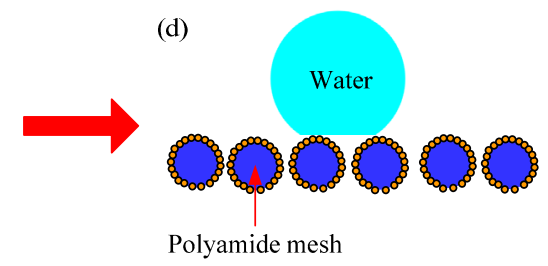

Fig. 1 Schematic illustration of superhydrophobic structure with controllable microstructure and mechanical robustness: (a) the flat surface with partially-embedded nanoparticles; (b) micro-protrusions with floating nanoparticles; (c) the micro-protrusions with partially-embedded nanoparticles; (d) controllable meshes with partially-embedded nanoparticles.

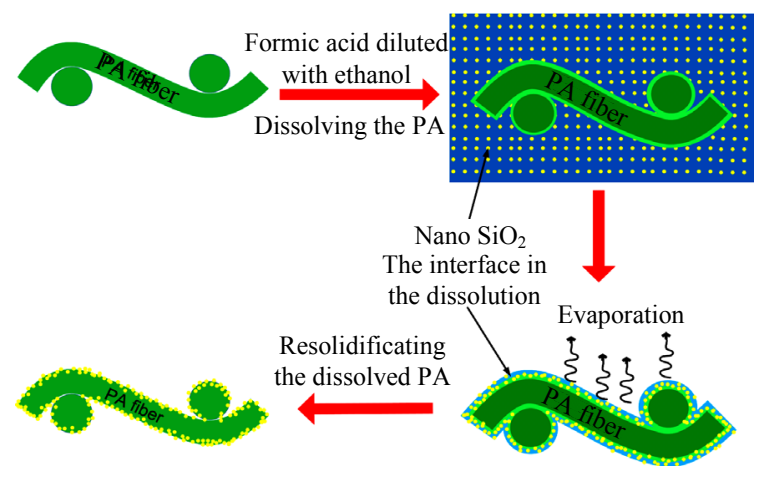

Fig. 2 The mechanism illustration of partially-embedding silica nanoparticles into the PA mesh.

droplets are suspended on but do not penetrate the micro/nano grooves of the surface as described by the Cassie-Baxter state ${ }^{[25,26]}$.

\subsection{The anti-icing test}

Superhydrophobic surfaces are one of the most promising candidate surfaces for passive anti-icing applications due to the existence of air pocket trapped in the solid-liquid interface as described by the CassieBaxter state. Ice adhesion is a crucial indicator for assessing the anti-icing property. Here, we tried to optimize ice adhesion by altering the mesh size of the PA mesh. As shown in Fig. 4a, only the fabric part contacted the ice when the superhydrophobic mesh was used. Thus, the areal fraction of the mesh $\left(f_{\mathrm{m}}\right)$ was further calculated using ImageJ software to represent the actual contact area of the mesh. The relationship between the adhesion strength and the mesh areal fraction $f_{\mathrm{m}}$ can be found in Fig. 4b. It could be found that the ice adhesion strength decreased as the mesh area fraction $f_{\mathrm{m}}$ decreased. As the Cassie-Baxter state described, the water droplets are suspended on the mesh fabrics (Fig. 5a). Thus, the smaller $f_{\mathrm{m}}$ value led to more air being trapped between the ice and mesh. The smallest adhesion strength was found to be $\sim 1.90 \mathrm{kPa}$ when the 60 -mesh sample was utilized, which is comparable to that of the well-textured superhydrophobic surface ${ }^{[12]}$. Tiny differences could be found between the 60-, 80-, and 100-mesh samples. Because of gravity, the contact line between the air and water tended to decrease as the spacing between the fabrics increased, and then the contact area increased (Fig. 5a).

Moreover, the adhesion strength increased dramatically when the mesh size reached to 400-mesh, which corresponded to a fraction of $f_{\mathrm{m}}=0.891$. Remarkably, the adhesion strength in the 500-mesh sample $\left(f_{\mathrm{m}}=0.987\right)$ was approximately 6 times higher than that of the 60 -mesh sample $\left(f_{\mathrm{m}}=0.638\right)$. Furthermore, the adhesion strength of the flat sample $\left(f_{\mathrm{m}}=1\right)$ is $\sim 1.6$ times larger than that of the 500-mesh sample $\left(f_{\mathrm{m}}=0.987\right)$. We attributed this to the microscale roughness of the 500-mesh sample, which could induce local stress concentrations, and significantly decrease the work required to remove ice from the surface ${ }^{[12,27]}$.

The stability of the ice adhesion strength is crucial for practical applications. Kulinich et al. found that the 

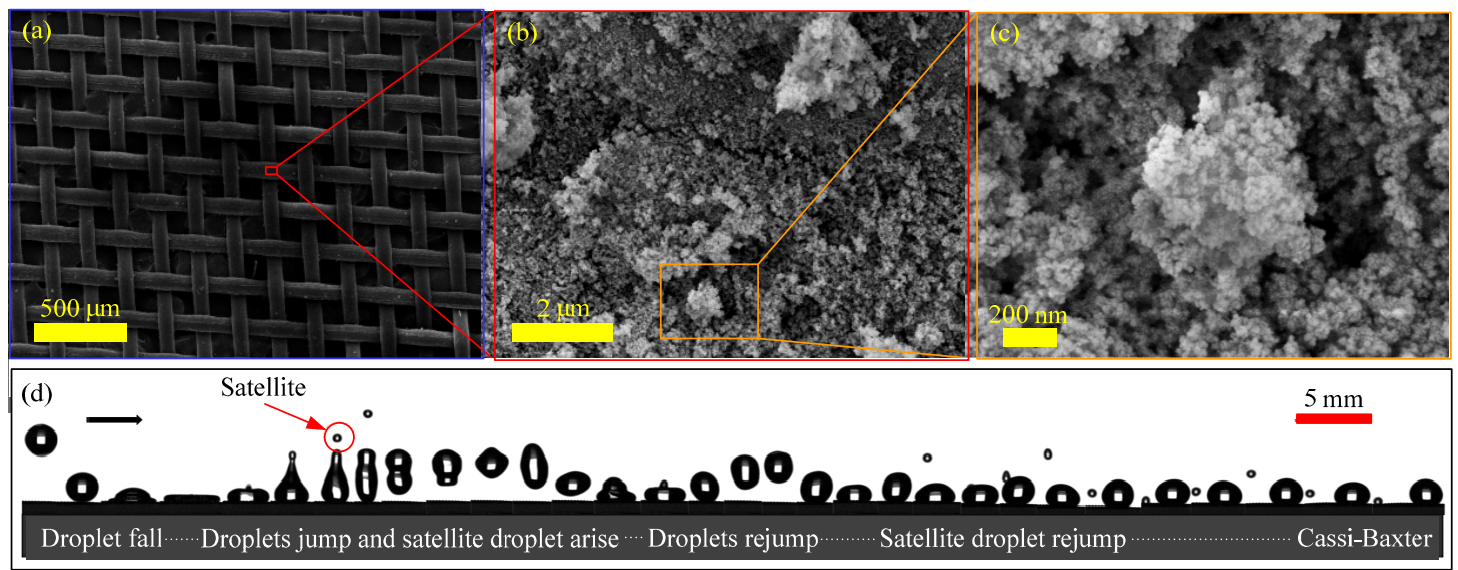

Fig. 3 (a - c) SEM images of a typical superhydrophobic mesh at different magnifications. (d) The images illustrate a water droplet bouncing on a superhydrophobic mesh.
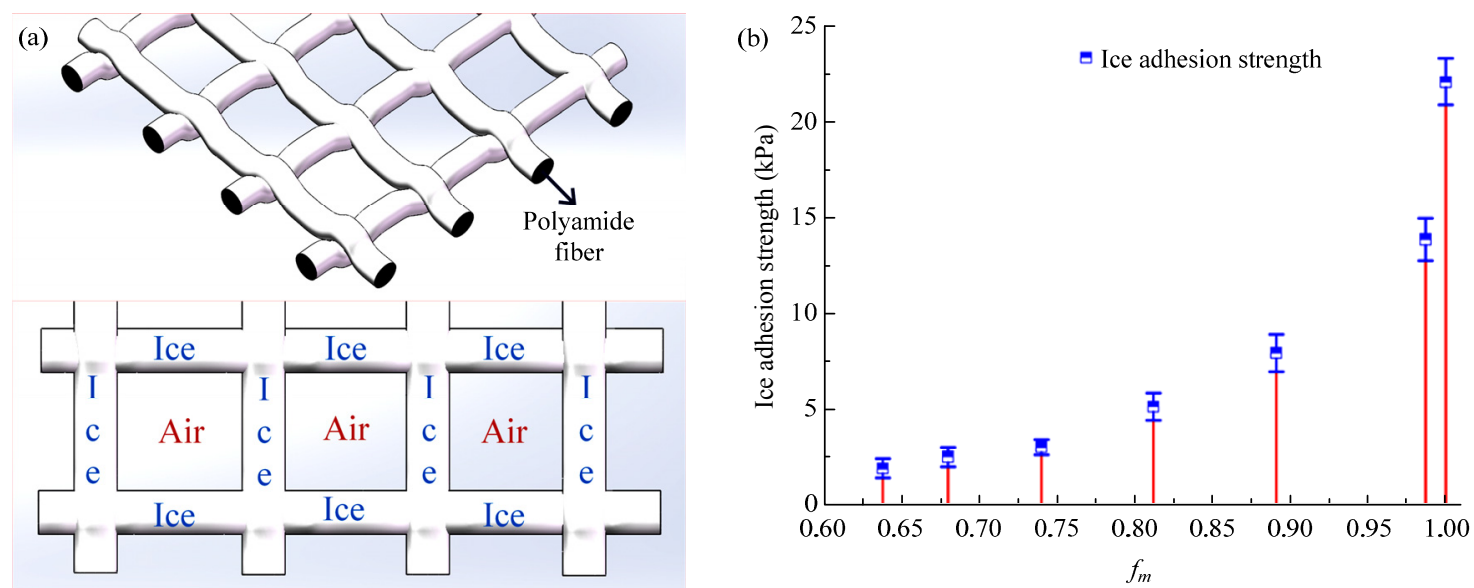

Fig. 4 (a) The structure illustration of the actual contact area for the polyamide mesh. (b) The relationship between ice adhesion strength and areal fraction $f_{\mathrm{m}}$.
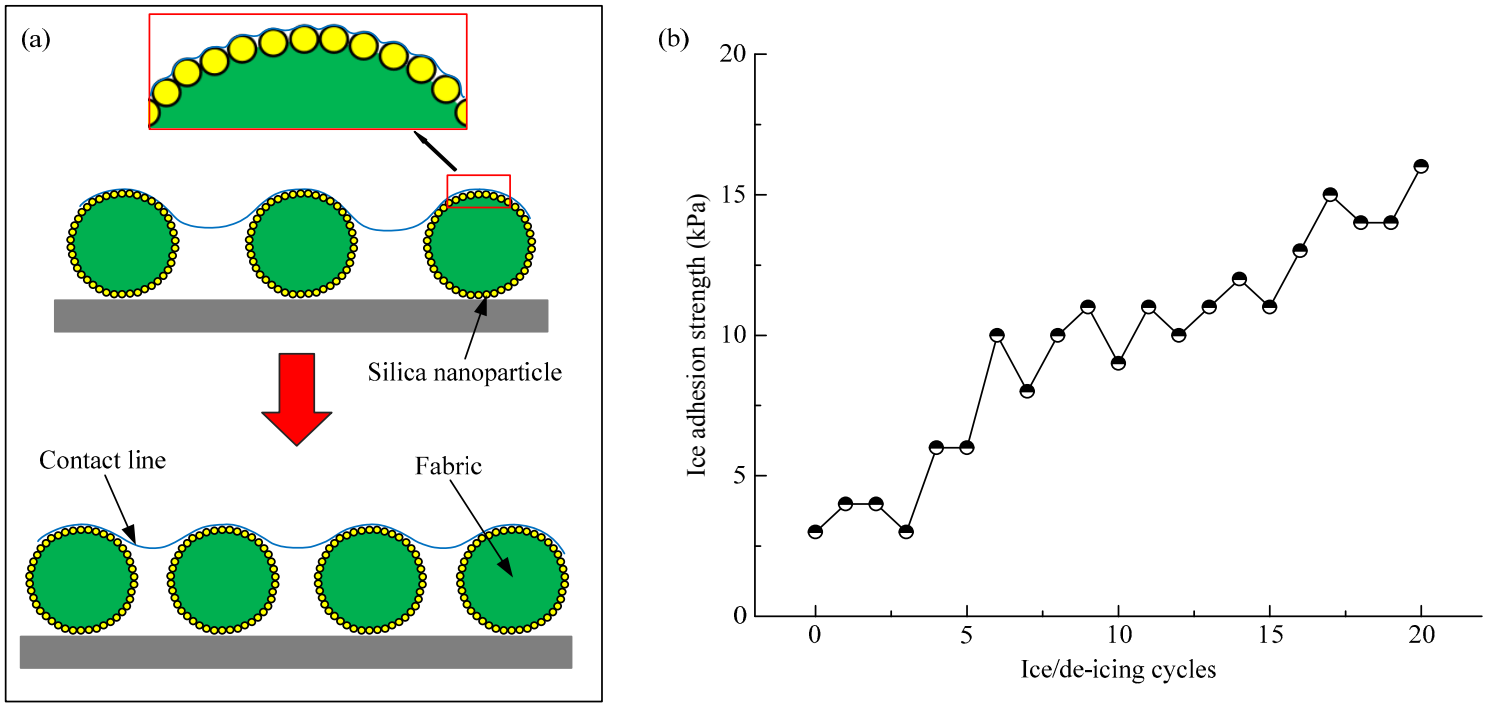

Fig. 5 (a) The illustration of the relationship between the contact line and the area fraction. (b) The plot of the ice adhesion strength as a function of icing-deicing cycles. 
surface asperities of superhydrophobic materials tended to be gradually damaged, which led to the loss of ice-repellence ${ }^{[15]}$. In this research, repetitive icing/deicing tests were performed. In our initial experiment, the polyamide meshes with different mesh sizes (60-, 80-, 100-, 300-, 400- and 500-mesh) were utilized in the icing/deicing test, and the 100-mesh sample demonstrated a better cyclic robustness than the other samples. The experimental results are summarized in Fig. 5b. It can be found that the adhesion strength gradually increased from $\sim 3 \mathrm{kPa}$ to $\sim 16 \mathrm{kPa}$. We conceded that the destruction of the surface asperity was inevitable. However, the ice adhesion strength is still lower that $20 \mathrm{kPa}$ after $20 \mathrm{icing} /$ deicing cycles. We attributed this to the combination of the hierarchical structure and partially-embedded structure. On the one hand, the microscale of the polyamide fabric could protect the nanoparticles from being abraded. On the other hand, the nanoparticles were partially embedded into the substrate, and then the substrate could offer physical support to the nanoparticles.

To further investigate the icephobicity, we tried to tightly attach the samples to a Peltier cooler, and controlled the surface temperature of the sample to $-15{ }^{\circ} \mathrm{C}$. Here, the superhydrophobic 100-mesh sample was utilized, with the superhydrophobic plate and glass plate as the reference sample. Then, water droplets $(\sim 5 \mu \mathrm{L})$ were dropped onto the surfaces to measure how long it takes for water droplets to be completely frozen. As shown in Fig. 6c, the water droplet on the bare glass plate exhibited a hemispherical shape, and it was the first to be completely frozen after $\sim 142 \mathrm{~s}$. The water droplets on both the Superhydrophobic Plate (SP) and Superhydrophobic Mesh (SM) demonstrated a nearly round shape. The water droplet on the SP surface was the second to be completely frozen after $\sim 316 \mathrm{~s}$ (Fig. 6b). Finally, the water droplet on the SM surface was completely frozen after $\sim 1048 \mathrm{~s}$ (Fig. 6a), indicating that the SM can significantly delay the freezing time. Moreover, the droplet maintained a round shape even after it was completely frozen. We further restored the temperature of the as-prepared surface to room temperature. Then the droplets on the three samples could be basically recovered.

The freezing delay property of the SM could be explained based on the heat transfer rate between the water droplet and the substrate as well as the dissipation of heat to the environment by convection ${ }^{[28,29]}$. The heat transfer rate by conduction between two surfaces can be described by the following Eq. (1 $)^{[30]}$ :

$$
\frac{\mathrm{d} Q}{\mathrm{~d} t}=-k A\left(\frac{\mathrm{d} T}{\mathrm{~d} x}\right)
$$

where $\mathrm{d} Q / \mathrm{d} t$ is the heat transfer rate $\left(\mathrm{J} \cdot \mathrm{s}^{-1}\right), k$ is the thermal conductivity coefficient $\left(\mathrm{J} \cdot \mathrm{m}^{-1} \cdot \mathrm{s}^{-1} \cdot \mathrm{k}^{-1}\right), A$ is the interfacial area $\left(\mathrm{m}^{2}\right)$, and $\mathrm{d} T / \mathrm{dx}$ is the temperature gradient. Considering this equation, superhydrophobic meshes in the Cassie-Baxter state tend to have low heat transfer rates. First, superhydrophobic surfaces have smaller interfacial area with sitting droplets ( $A$ in Eq. (1)) compared to low contact angle surfaces. It has been reported that the fraction of contact between a liquid and substrate in superhydrophobic surfaces is less than $10 \%{ }^{[31,32]}$. This low interfacial surface area leads to a lower heat transfer rate ${ }^{[27-29]}$. Second, an air layer exists between the droplet and the substrate in the CassieBaxter state and acts as an insulator to lower the heat transfer rate ${ }^{[33]}$. This rule is more obvious on this superhydrophobic mesh, as the SM exhibited a much smaller interfacial area than the SP due to its structure (Fig. 4a).

\subsection{The mechanical and chemical robustness test}

Besides the anti-icing property, the mechanical robustness was the Achilles' heel of superhydrophobicity. Here, the sandpaper-abrasion test was employed to assess the mechanical robustness as this test is the most widely used method. Tian et al. further claimed that the applied pressure and abrasion distance are two crucial indicators for comparing different researches ${ }^{[34]}$. In this research, polyamide meshes with different mesh sizes (60-, 80-, 100-, 300, 400-, and 500-mesh) were utilized in the sandpaper abrasion test. It was found that the 100-mesh sample exhibits a better anti-abrasion ability than the other samples, and this results was similar to that of the icing-deicing test. Here, we applied a much larger pressure of $12.5 \mathrm{kPa}$ (500 g weight, as shown in Fig. 7a) than the other studies ${ }^{[35-45]}$. As shown in supplementary movie 7 and Fig. 7a, the sample was placed face down on the rough surface of SiC sandpaper (1200-mesh), and moved $20 \mathrm{~cm}$ along the ruler for 
(a)

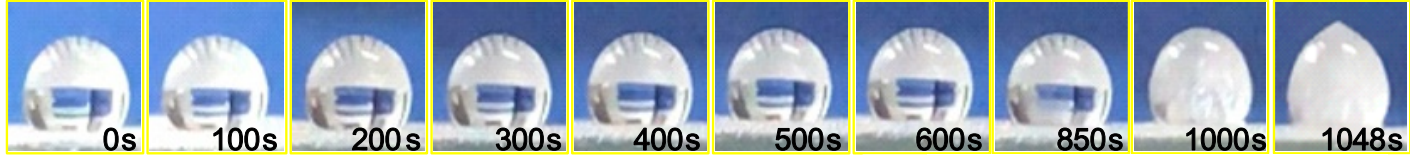

(b)

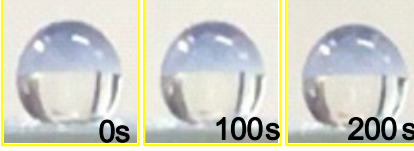

(c)

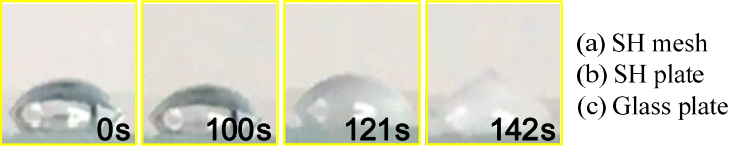

(d) $316 s$

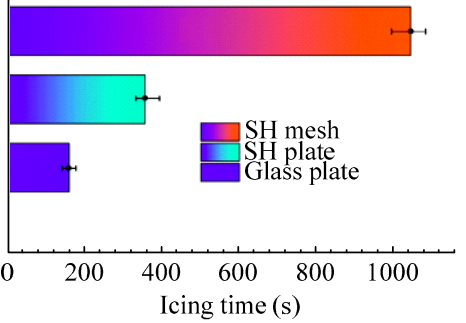

Fig. 6 The images that exhibit the freezing process of a water droplet on a (a) superhydrophobic (SH) mesh, (b) superhydrophobic plate, and (c) bare glass plate. (d) The comparison of the icing time of the $\mathrm{SH}$ mesh, $\mathrm{SH}$ plate, and glass plate.

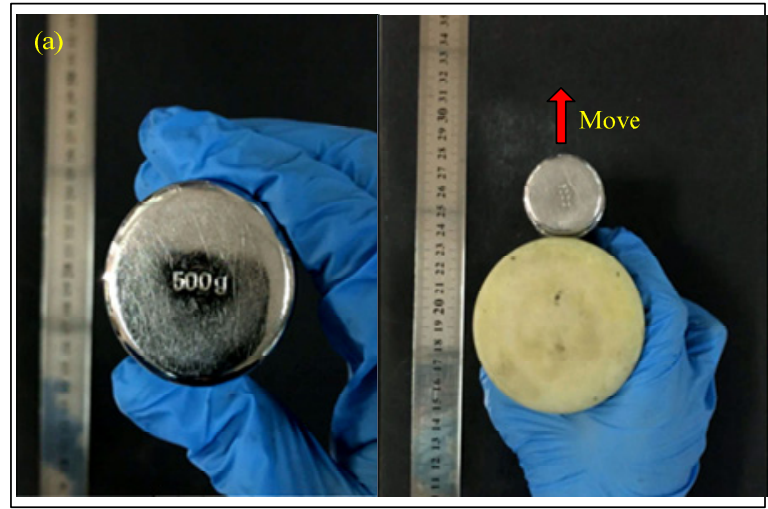

(c)
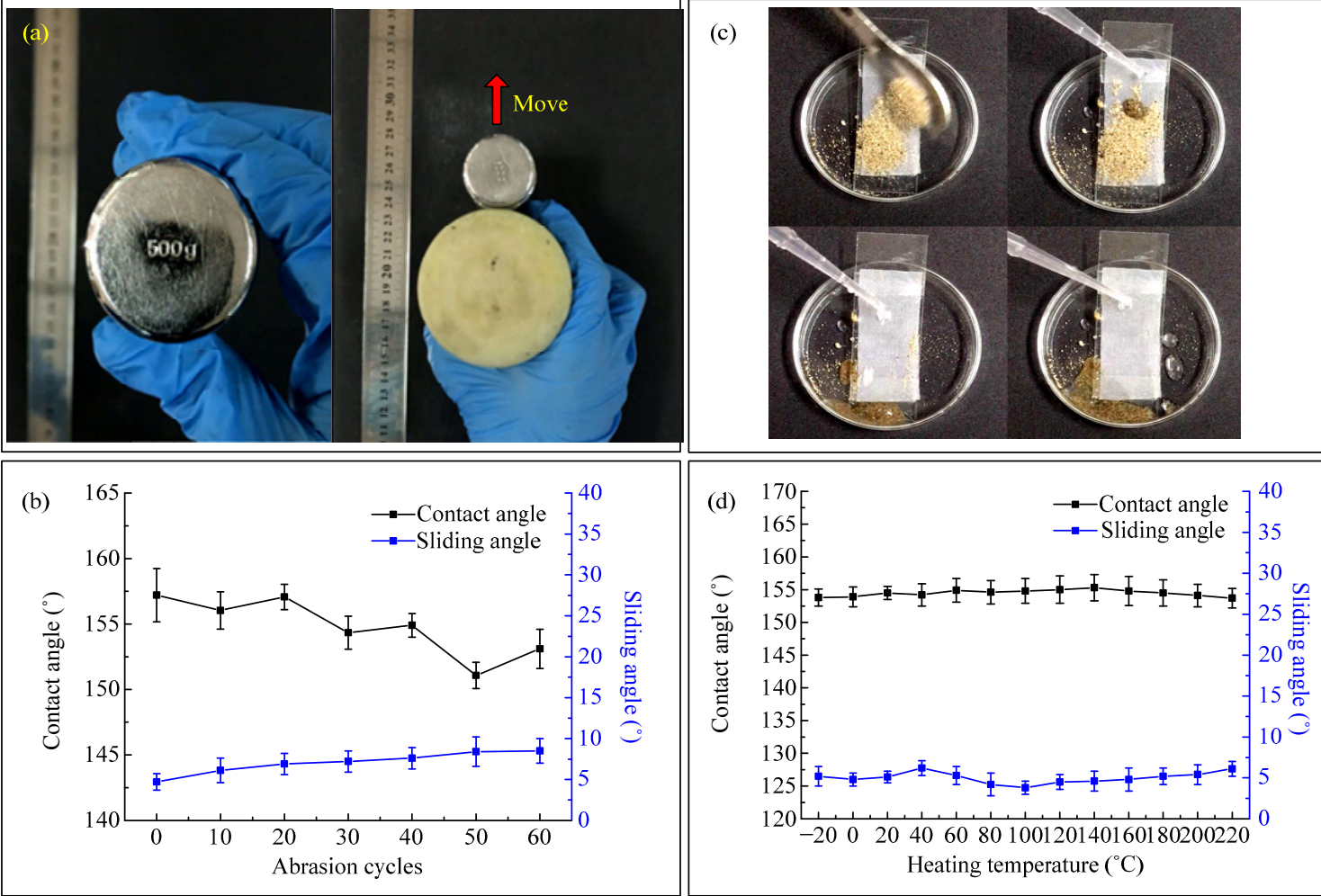

Fig. 7 (a) The image of the sandpaper-abrasion process. (b) The plot of the CAs and SAs as a function of the number of abrasion cycles. (c) The images demonstrating the self-cleaning process. (d) The plot of the CAs and SAs after the samples were heated at different temperatures for $24 \mathrm{~h}$.

one abrasion cycle. Although some powders appeared during the abrasion process, the behaviour of the water droplets indicated that superhydrophobicity was maintained (see supplementary movie 7).

We further quantitatively measured the changes in the CAs and SAs during the abrasion process (Fig. 7b). The CA was $153.1^{\circ}$ and the SA was $8.5^{\circ}$ even after 60 abrasion cycles. For the convenience of comparison, we also utilized a relatively low pressure of $2.5 \mathrm{kPa}(100 \mathrm{~g}$ weight) to abrade the sample, and the sample retained superhydrophobicity even after 450 cycles $(90.0 \mathrm{~m})$. The comprehensive comparison of our work with other results can be found in Table 1. Thus, it can be concluded that this superhydrophobic mesh demonstrated excellent 
Table 1 Comparison of the anti-abrasion ability of the superhydrophobic mesh with that of previously reported works

\begin{tabular}{|c|c|c|c|}
\hline Material & Applied Load & Distance & Ref. \\
\hline $\mathrm{TiO}_{2} \mathrm{NPs}+$ adhesive & $100 \mathrm{~g}$ & $8.00 \mathrm{~m}$ & {$[16]$} \\
\hline PDMS@ZHS & $100 \mathrm{~g}$ & $3.00 \mathrm{~m}$ & {$[35]$} \\
\hline Concrete/sand/fluoralkylsilane & $1.10 \mathrm{kPa}$ & $8.00 \mathrm{~m}$ & {$[36]$} \\
\hline NPs + inorganic glue & $200 \mathrm{~g}$ & $10.00 \mathrm{~m}$ & {$[37]$} \\
\hline Silicone resin and silica sol & $2.67 \mathrm{kPa}$ & $0.80 \mathrm{~m}$ & {$[38]$} \\
\hline UHMWPE/SiO2/NiO & $200 \mathrm{~g}(2.25 \mathrm{kPa})$ & $9.65 \mathrm{~m}$ & {$[39]$} \\
\hline PU spray + silanized palygorskite & $2.3 \mathrm{kPa}$ & $80.00 \mathrm{~m}$ & {$[40]$} \\
\hline NPs and polystyrene & $500 \mathrm{~g}(7.84 \mathrm{kPa})$ & $6.75 \mathrm{~m}$ & {$[41]$} \\
\hline Hierarchical Si structure & $3.45 \mathrm{kPa}$ & $0.25 \mathrm{~m}$ & {$[42]$} \\
\hline $\mathrm{PDMS} / \mathrm{TiO}_{2} \mathrm{NPs}$ & $100 \mathrm{~g}$ & $5.00 \mathrm{~m}$ & {$[43]$} \\
\hline Polyamide mesh $/ \mathrm{SiO}_{2} \mathrm{NPs}$ & $\begin{array}{c}100 \mathrm{~g}(2.5 \mathrm{kPa}) \\
500 \mathrm{~g}(12.5 \mathrm{kPa})\end{array}$ & $\begin{array}{l}90.00 \mathrm{~m} \\
12.00 \mathrm{~m}\end{array}$ & \\
\hline
\end{tabular}

mechanical robustness.

Moreover, this superhydrophobic mesh displays a superior self-cleaning property. Here, we utilized the soil as the model contaminant. As shown in supplementary movie 8 and Fig. 7c, the water droplets rolled down easily on the superhydrophobic mesh, and tended to carry the contacted contaminant away. After the water dropping process, a completely clean sample was obtained, indicating the superior self-cleaning performance. Thermal stability is also an essential indicator for assessing superhydrophobic materials' environmental adaptability. As shown in Fig. 7d, the CAs and SAs of the superhydrophobic mesh were measured from $-20{ }^{\circ} \mathrm{C}$ to $220{ }^{\circ} \mathrm{C}$ for $24 \mathrm{~h}$. Remarkably, the changes in both the CAs and SAs were very small which suggests the excellent thermostability.

\section{Conclusion}

In summary, a passive anti-icing strategy based on superhydrophobic mesh was reported. A polyamide mesh was utilized as the substrate due to its cost-efficiency and excellent machinability. We tried to partially embed silica nanoparticles into micro-scale fabrics of a commercial polyamide mesh. Note that the areal fraction of the polyester mesh could be determined by altering the mesh size in the local market. Then, we studied the contribution of the area fraction to the ice adhesion strength. Generally, the ice adhesion strength decreases as the area fraction decreases. It was found that the combination of superhydrophobicity and stress concentration led to the low ice adhesion strength. The minimum ice adhesion strength can be as small as $\sim 1.9 \mathrm{kPa}$, and the delayed freezing time can be as long as $\sim 1048$ s. Furthermore, the combination of a partially-embedded structure and a hierarchical structure endows this superhydrophobic mesh with excellent robustness. Then this superhydrophobic mesh could withstand $20 \mathrm{icing} /$ deicing cycles, 60 sandpaper abrasion cycles and a $220{ }^{\circ} \mathrm{C}$ temperature treatment. This superhydrophobic mesh not only can be used alone just like a screen window, but also can cover surfaces to be protected with the help of double-sided tape/glue. Thus, this superhydrophobic mesh might have a bright future in the application for anti-icing.

\section{Acknowledgment}

This work was supported by National Nature Science Foundation of China (51977079, 51607067), Youth Elite Scientists Sponsorship Program by Chinese Society for Electrical Engineering (CSEE-YESS -2017002), and the Fundamental Research Funds for the Central Universities (2020MS115, 2017MS149).

* All supplementary materials are available at https://doi.org/10.1007/s42235-021-0012-4.

Open Access This article is licensed under a Creative Commons Attribution 4.0 International License, which permits use, sharing, adaptation, distribution and reproduction in any medium or format, as long as you give appropriate credit to the original author(s) and the source, provide a link to the Creative Commons licence, and indicate if changes were made.

The images or other third party material in this article are included in the article's Creative Commons licence, unless indicated otherwise in a credit line to the material. If material is not included in the article's Creative Commons licence and your intended use is not permitted by statutory regulation or exceeds the permitted use, you will need to obtain permission directly from the copyright holder.

To view a copy of this licence, visit http://creativecommons.org/licenses/by/4.0/.

\section{References}

[1] Golovin K, Dhyani A, Thouless M D, Tuteja A. Low-interfacial toughness materials for effective large-scale deicing. Science, 2019, 364, 371-375. 
[2] Liu W L, Chen H F, Shen Y Z, Wu Z W. Facilely fabricating superhydrophobic resin-based coatings with lower water freezing temperature and ice adhesion for anti-icing application. Journal Bionic Engineering, 2019, 16, 794-805.

[3] Wang G W, Guo Z G. Liquid infused surfaces with anti-icing properties. Nanoscale, 2019, 11, 22615-22635.

[4] Liao R J, Zuo Z P, Guo C, Zhuang A Y, Yuan Y, Zhao X T, Zhang Y Y. Ice accretion on superhydrophobic insulators under freezing condition. Cold Regions Science and Technology, 2015, 112, 87-94.

[5] Latthe S S, Sutar R S, Bhosale A K, Nagappan S, Ha C S, Sadasivuni K K, Liu S, Xing R M. Recent developments in air-trapped superhydrophobic and liquid-infused slippery surfaces for anti-icing application. Progress in Organic Coatings, 2019, 137, 105373.

[6] Chu Z M, Jiao W C, Huang Y F, Ding G M, Zhong X, Yan M L, Zheng Y T, Wang R G. FDTS-modified $\mathrm{SiO}_{2} / \mathrm{rGO}$ wrinkled films with a micro-nanoscale hierarchical structure and anti-icing/deicing properties under condensation condition. Advanced Materials Interfaces, 2019, 7, 1901446.

[7] Zhang X M, Fu F, Gao X M, Hou X F. Magnetically driven superhydrophobic polyurethane sponge for high efficiency oil/water mixtures separation. Journal of Bionic Engineering, 2019, 16, 38-46.

[8] Xu X H, Zhang Z Z, Guo F, Jin Yang, Zhu X T, Zhou X Y, Xue Q J. Fabrication of bionic superhydrophobic manganese oxide/polystyrene nanocomposite coating. Journal of Bionic Engineering, 2012, 9, 11-17.

[9] Wang P, Wang S, Zhang X M, Wang H Q, Duan W, Han H L, Fan X L. Rational construction of $\mathrm{CoO} / \mathrm{CoF}_{2}$ coating on burnt-pot inspired 2D CNs as the battery-like electrode for supercapacitors. Journal of Alloys and Compounds, 2020, 819, 153374.

[10] Wang P, Wei W D, Li Z Q, Duan W, Han H L, Xie Q. A superhydrophobic fluorinated PDMS composite as a wearable strain sensor with excellent mechanical robustness and liquid impalement resistance. Journal of Materials Chemistry A, 2020, 8, 3509-3516.

[11] Beemer D L, Wang W, Kota A K. Durable gels with ultra-low adhesion to ice. Journal of Materials Chemistry A, 2016, 4, 18253-18258.

[12] Xie Y H, Chen H F, Shen Y Z, Tao J, Jin M M, Wu Y, Hou W Q. Rational fabrication of superhydrophobic nanocone surface for dynamic water repellency and anti-icing potential. Journal of Bionic Engineering, 2019, 16, 27-37.

[13] Pan R, Cai M Y, Liu W J, Luo X, Chen C H, Zhang H J, Zhong M L. Extremely high Cassie-Baxter state stability of superhydrophobic surfaces via precisely tunable dual-scale and triple-scale micro-nano structures. Journal of Materials Chemistry A, 2019, 7, 18050-18062.

[14] Ling E J Y, Uong V, Renault-Crispo J, Kietzig A, Servio P, Reducing ice adhesion on nonsmooth metallic surfaces: Wettability and topography effects. ACS Applied Materials \& Interfaces, 2016, 8, 8789-8800.

[15] Kulinich S A, Farhadi S, Nose K, Du X W. Superhydrophobic surfaces: are they really ice-repellent? Langmuir, 2011, 27, 25-29.

[16] Lu Y, Sathasivam S, Song J, Crick C R, Carmalt C J, Parkin I P. Robust self-cleaning surfaces that function when expose to either air or oil. Science, 2015, 347, 1132-1135.

[17] Verho T, Bower C, Andrew P, Franssila S, Ikkala O, Ras R H A. Mechanically durable superhydrophobic surfaces. Advanced Materials, 2011, 23, 673-678.

[18] Wang P, Sun B, Yao T, Chen M J, Fan X L, Han H L, Li L, Wang C. A novel dissolution and resolidification method for preparing robust superhydrophobic polystyrene/silica composite. Chemical Engineering Journal, 2017, 326, 1066-1073.

[19] Nguyen T B, Park S, Lim H. Effects of morphology parameters on anti-icing performance in superhydrophobic surfaces. Applied Surface Science, 2018, 435, 585-591.

[20] Yokoi N, Manabe K, Tenjimbayashi M, Shiratori S. Optically transparent superhydrophobic surfaces with enhanced mechanical abrasion resistance enabled by mesh structure. ACS Applied Materials \& Interfaces, 2015, 7, 4809-4816.

[21] Meuler A J, Smith J D, Varanasi K K, Mabry J M, McKinley $\mathrm{G} \mathrm{H}$, Cohen R E. Relationships between water wettability and ice adhesion. ACS Applied Materials \& Interfaces, 2010, 2, 3100-3110.

[22] Jiang G, Chen L, Zhang S D, Huang H X. Superhydrophobic $\mathrm{SiC} / \mathrm{CNT}$ s coatings with photothermal deicing and passive anti-icing properties. ACS Applied Materials \& Interfaces, 2018, 10, 36505-36511.

[23] Wang P, Chen M J, Han H L, Fan X L, Liu Q, Wang J F. Transparent and abrasion-resistant superhydrophobic coating with robust self-cleaning function in either air or oil. Journal of Materials Chemistry A, 2016, 4, 7869-7874.

[24] Wang S D, Luo S S. Fabrication of transparent superhydrophobic silica-based film on a glass substrate. Applied Surface Science, 2012, 258, 5443-5450.

[25] Wang N, Xiong D S, Deng Y L, Shi Y, Wang K. Mechanically robust superhydrophobic steel surface with anti-icing, UV-durability, and corrosion resistance properties. ACS Applied Materials \& Interfaces, 2015, 7, 6260-6272. 
[26] Wang X Y, Li M J, Shen Y Q, Yang Y X, Feng H, Li J. Facile preparation of loess-coated membranes for multifunctional surfactant-stabilized oil-in-water emulsion separation. Green Chemistry, 2019, 21, 3190-3199.

[27] Liu Y, Li X L, Jin J F, Jin J F, Liu J A, Yan Y Y, Han Z W, Ren L Q. Anti-icing property of bio-inspired micro-structure superhydrophobic surface and heat transfer model. Applied Surface Science, 2017, 400, 498-505.

[28] Alizadeh A, Yamada M, Li R, Shang W, Otta S, Zhong S, Ge L. Dynamics of ice nucleation on water repellent surfaces. Langmuir, 2012, 28, 3180-3186.

[29] Jung S, Tiwari M K, Doan N V, Poulikakos D. Mechanism of supercooled droplet freezing on surfaces. Nature Coтmunications, 2012, 3, 615.

[30] Boreyko J B, Collier C P. Delayed frost growth on jumping-drop superhydrophobic surfaces. ACS Nano, 2013, 7, 1618-1627.

[31] Sarshar M A, Song D, Swarctz C, Lee J, Choi C H. Anti-icing or deicing: Icephobicities of superhydrophobic surfaces with hierarchical structures. Langmuir, 2018, 34, 13821-13827.

[32] Qi C H, Chen H, Shen L Y, Li X L, Fu Q, Zhang Y H, Sun Y Y, Liu Y Q. Superhydrophobic surface based on assembly of nanoparticles for application in anti-icing under ultralow temperature. ACS Applied Nano Materials, 2020, 3, 2047-2057.

[33] Muster T H, Prestidge C A, Hayes R A. Water adsorption kinetics and contact angles of silica particles. Colloids and Surfaces A: Physicochemical and Engineering, 2001, 176, 253-266.

[34] Tian X L, Verho T, Ras R H A. Moving superhydrophobic surfaces toward real-world applications. Science, 2016, 352, 142-143.

[35] Long M Y, Peng S, Deng W S, Miao X R, Wen N, Zhou Q N, Yang $\mathrm{X}$ J, Deng $\mathrm{W}$ L. A robust superhydrophobic PDMS@ZnSn $(\mathrm{OH})_{6}$ coating with under-oil self-cleaning and flame retardancy. Journal of Materials Chemistry A,
2017, 5, 22761-22771.

[36] Song J L, Zhao D Y, Han Z J, Xu W, Lu Y, Liu X, Liu B, Carmalt C J, Deng X, Parkin I P. Super-robust superhydrophobic concrete. Journal of Materials Chemistry A, 2017, 5, 14542-14550.

[37] Liu M M, Li J, Hou Y Y, Guo Z G. Inorganic adhesives for robust superwetting surfaces. ACS Nano, 2017, 11, 1113-1119.

[38] Chen L, Sun X Y, Hang J Z, Jin L J, Shang D, Shi L Y. Large-scale fabrication of robust superhydrophobic coatings with high rigidity and good flexibility. Advanced Materials Interfaces, 2016, 3, 1500718.

[39] Wang N, Lu Y, Xiong D S, Carmalt C J, Parkin I P. Designing durable and flexible superhydrophobic coatings and its application in oil purification. Journal of Materials Chemistry A, 2016, 4, 4107-4116.

[40] Zhang J P, Gao Z Q, Li L X, Li B C, Sun H X. Waterborne nonfluorinated superhydrophobic coatings with exceptional mechanical durability based on natural nanorods. Advanced Materials Interfaces, 2017, 4, 1700723.

[41] Cai Z M, Shen L, Wang X J, Guo Q P. Fabrication of a bulk superhydrophobic conductive material by mechanical abrasion. Composite Science Technology, 2018, 164, 238-247.

[42] Xiu Y H, Liu Y, Hess D W, Wong C. Mechanically robust superhydrophobicity on hierarchically structured Si surfaces. Nanotechnology, 2010, 21, 155705.

[43] Hoshian S, Jokinen V, Franssila S. Robust hybrid elastomer/metal-oxide superhydrophobic surfaces. Soft Matter, 2016, 15, 6526-6535.

[44] Chen F Z, Song J L, Lu Y, Huang S, Liu X, Sun J, Carmalt C J, Parkin I P, Xu W J, Creating robust superamphiphobic coatings for both hard and soft materials. Journal of Materials Chemistry A, 2015, 3, 20999-21008.

[45] Wang P, Yao T, Li Z Q, Wei W D, Xie Q, Duan W, Han H L, A superhydrophobic/electrothermal synergistically anti-icing strategy based on graphene composite. Composites Science and Technology, 2020, 198, 108307. 